\title{
Fokker-Planck equations and density of states in disordered quantum wires
}

\author{
M. Titov, ${ }^{1}$ P. W. Brouwer, ${ }^{2}$ A. Furusaki, ${ }^{3}$ and C. Mudry ${ }^{4}$ \\ ${ }^{1}$ Instituut-Lorentz, Universiteit Leiden, P.O. Box 9506, 2300 RA Leiden, The Netherlands \\ ${ }^{2}$ Laboratory of Atomic and Solid State Physics, Cornell University, Ithaca, NY 14853-2501 \\ ${ }^{3}$ Yukawa Institute for Theoretical Physics, Kyoto University, Kyoto 606-8502, Japan \\ ${ }^{4}$ Paul Scherrer Institut, CH-5232 Villigen PSI, Switzerland
}

(Received 8 November 2000; revised manuscript received 17 January 2000; published 30 May 2001)

\begin{abstract}
We propose a general scheme to construct scaling equations for the density of states in disordered quantum wires for all ten pure Cartan symmetry classes. The anomalous behavior of the density of states near the Fermi level $\varepsilon=0$ for the three chiral and four Bogoliubov-de Gennes universality classes is analyzed in detail by means of a mapping to a scaling equation for the reflection from a quantum wire in the presence of an imaginary potential.
\end{abstract}

DOI: 10.1103/PhysRevB.63.235318

PACS number(s): 73.23.-b, 71.55.Jv, 71.23.-k, 72.15.Rn

\section{INTRODUCTION}

Statistical properties of energy levels and wave functions in disordered electron systems are believed to be determined by, first of all, the fundamental symmetries of the Hamiltonian. Such a connection is best established in the case of "'zero dimension," i.e., for finite-size systems with a large dimensionless conductance $g$, where a description in terms of random matrix theory is valid. Using the link to Cartan's classification of symmetric spaces, ${ }^{1}$ Zirnbauer $^{2}$ and Caselle ${ }^{3}$ have pointed out that there exist only ten possible random matrix theories, whose form follows directly from the geometrical characteristics ("roots") of the corresponding symmetric space in Cartan's table. These ten random matrix theories are divided into three standard classes, ${ }^{4}$ three chiral classes, ${ }^{5}$ and four Bogoliubov-de Gennes (BdG) classes, ${ }^{6}$ the subdivision in each class depending on the presence or absence of time-reversal symmetry (TR) and spin-rotation invariance $(\mathrm{SR}){ }^{7}$ see Table I.

The rational for the Cartan classification is believed to transcend "zero dimension" and has been applied to the construction of effective theories (e.g., nonlinear $\sigma$ models) for higher-dimensional disordered systems. The standard classes are thus believed to be appropriate to the problem of an electron moving in a random potential, without further symmetries, ${ }^{8}$ irrespective of dimensionality. The chiral classes are appropriate to the case when the disorder is purely "off-diagonal,", as is the case, e.g., for the lattice random flux model in quasi-one- and two dimensions, ${ }^{10,11}$ the random hopping model, ${ }^{12,13}$ and for random $X Y$ spin chains. ${ }^{14}$ The BdG classes refer to systems with superconducting correlations, ${ }^{15}$ and were argued to be valid for vortices in superconductors, ${ }^{16,17}$ dirty unconventional superconductors, ${ }^{18,19}$ and (in the case of broken time-reversal

TABLE I. Classification of symmetry classes according to Cartan's table. The symmetry classes are defined in terms of the presence or absence of time-reversal symmetry (TR) and spin-rotation invariance (SR), and in terms of the other fundamental symmetries of the system (standard, chiral, or BdG). The table further contains the multiplicities of the ordinary and long roots $m_{o}$ and $m_{l}$ and the degeneracy $d$ of the reflection and/or transmission eigenvalues (Ref. 25). In the seventh column we denote, using Cartan's notation (Chap. X of Ref. 1), the symmetric spaces associated with the transfer matrix group $\mathcal{M}$ of the quantum wire; these are different from the symmetric spaces associated with the microscopic Hamiltonian $\mathcal{H}$ of the quantum wire, which are listed in the last column. Following the convention of Ref. 6, we refer to the ten symmetry classes by Cartan's symbol for the symmetric space of their Hamiltonians. For standard and chiral quantum wires without TR the degeneracy $d$ of the reflection eigenvalues is 2 (1) with (without) SR.

\begin{tabular}{lccccccc}
\hline \hline Class & TR & SR & $m_{o}$ & $m_{l}$ & $d$ & $\mathcal{M}$ & $\mathcal{H}$ \\
\hline \multirow{4}{*}{ Standard } & Yes & Yes & 1 & 1 & 2 & CI & AI \\
& No & Yes (No) & 2 & 1 & $2(1)$ & AIII & A \\
& Yes & No & 4 & 1 & 2 & DIII & AII \\
\hline \multirow{4}{*}{ Chiral } & Yes & Yes & 1 & 0 & 2 & AI & BDI \\
& No & Yes (No) & 2 & 0 & $2(1)$ & A & AIII \\
& Yes & No & 4 & 0 & 2 & AII & CII \\
\hline \multirow{4}{*}{ BdG } & Yes & Yes & 2 & 2 & 4 & C & CI \\
& No & Yes & 4 & 3 & 4 & CII & C \\
& Yes & No & 2 & 0 & 2 & D & DIII \\
& No & No & 1 & 0 & 1 & BDI & D \\
\hline \hline
\end{tabular}




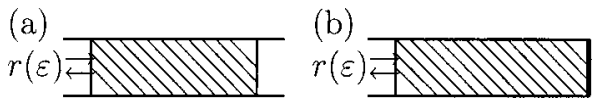

FIG. 1. A quantum wire (hashed marked region) of length $L$ connected to ideal leads. The reflection matrix at energy $\varepsilon, r(\varepsilon)$, is the matrix of reflection amplitudes for reflection into the same lead. (a) A two-lead geometry is used to compute the conductance. (b) A one-lead geometry is used to compute the DOS whereby the wire is closed by a perfectly reflecting wall on the right.

symmetry) normal metals in proximity to a superconductor. The BdG classes have also been argued to be of some relevance to problems in statistical mechanics such as random bond Ising and network models. ${ }^{20,21}$

The geometric structure of the symmetric spaces not only determines the level statistics in zero dimension, it also determines the form of scaling equations for the transmission eigenvalues $T_{j}$ and reflection eigenvalues $R_{j}=1$ $-T_{j}(j=1, \ldots, N)$ in a wire geometry, where a disordered sample of length $L$ is connected to two ideal leads [see Fig. 1(a).$^{3,22,23}$ Here the reflection eigenvalues $R_{1}, \ldots, R_{N}$ are the $d$-fold degenerate eigenvalues of the matrix product $r^{\dagger} r$, where $r$ is the $N d$-dimensional matrix of reflection amplitudes for backreflection into the same lead [see Fig. 1(a)] and $N d$ is the number of propagating channels at the Fermi level. The degeneracies $d$ are listed in Table I for the ten symmetry classes in Cartan's classification. Parametrizing

$$
R_{j}=\tanh ^{2} x_{j},
$$

the scaling equation for the distribution $P\left(x_{1}, \ldots, x_{N} ; L\right)$ takes the form ${ }^{3,24}$

$$
\frac{\partial P}{\partial L}=\frac{1}{2 \gamma l} \sum_{j=1}^{N} \frac{\partial}{\partial x_{j}} J \frac{\partial}{\partial x_{j}} J^{-1} P,
$$

where $l$ is the mean free path and $L$ is the length of the quantum wire. For the standard and BdG symmetry classes, the $x_{j}$ are positive random variables, $0<x_{j}<\infty$, and the Jacobian $J$ and the numerical constant $\gamma$ read

$$
\begin{gathered}
J=\prod_{j=1}^{N} \sinh ^{m_{l}}\left(2 x_{j}\right) \prod_{k<j} \prod_{ \pm} \sinh ^{m_{o}}\left(x_{j} \pm x_{k}\right), \\
\gamma=m_{o}(N-1)+m_{l}+1,
\end{gathered}
$$

while for the chiral classes the $x_{j}$ can take values on the entire real axis, $-\infty<x_{j}<\infty$, and one has

$$
\begin{gathered}
J=\prod_{j=1}^{N} \prod_{k<j} \sinh ^{m_{o}}\left(x_{j}-x_{k}\right), \\
\gamma=\frac{1}{2}\left[m_{o}(N-1)+2\right] .
\end{gathered}
$$

Here $m_{l}$ and $m_{o}$ are the multiplicities of the so-called long and ordinary roots for the corresponding symmetric space, ${ }^{25}$ respectively, see Table I. (For the chiral classes we only consider the case when the nonuniversality parameter $\eta$ equals unity, see Ref. 26.) For the standard classes, Eq. (2) was derived by Dorokhov, ${ }^{27}$ and Mello, Pereyra, and
Kumar; ${ }^{28}$ derivations for the chiral and $\mathrm{BdG}$ symmetry classes can be found in Refs. 29 and 30.

In this paper we show how the same structure also determines scaling equations for the density of states (DOS) $\nu(\varepsilon)$ in a quantum wire of infinite length $(L \rightarrow \infty)$ at energy $\varepsilon$. Our work builds on previous work for the chiral classes, where such a scaling equation was derived using a different method. ${ }^{31}$ In addition, we present an exact solution for the DOS, something that could not be done in Ref. 31 .

While $\nu(\varepsilon)$ is a nonsingular function of energy for the three standard classes, singular behavior is expected near $\varepsilon$ $=0$ for the remaining seven symmetry classes, as $\varepsilon=0$ is a special point there. (The energy $\varepsilon=0$ corresponds to a point of particle-hole symmetry in the chiral and BdG symmetry classes; it is the band center for lattice models with random hopping, or the Fermi energy in the case of the BdG classes.) Indeed, for the chiral classes, $\nu(\varepsilon)$ was found to depend sensitively on the parity of the channel number $N$ for $\varepsilon$ close to zero, showing the $\nu(\varepsilon) \propto 1 /\left|\varepsilon \ln ^{3} \varepsilon\right|$ divergence characteristic of pure one-dimensional systems with chiral symmetry for odd $N,{ }^{32}$ while $\nu(\varepsilon) \propto\left|\varepsilon^{m_{o}-1} \ln \varepsilon\right|$ for even $N{ }^{31}$ For the $\mathrm{BdG}$ classes in the presence of spin-rotation invariance (classes $\mathrm{C}$ and $\mathrm{CI}$; we refer to the symmetry classes by Cartan's symbol for the symmetric space corresponding to their Hamiltonians, see Table I), a suppression $\nu(\varepsilon) \propto \varepsilon^{m_{l}-1}$ is expected. ${ }^{18,33}$ In both cases, the characteristic energy scale for the DOS singularity is $\sim \hbar v_{F} / N^{2} l, v_{F}$ being the Fermi velocity. This distinguishes the singularity in $\nu(\varepsilon)$ for quantum wires from its counterpart in zero dimension, where the characteristic energy scale is the mean level spacing which goes to zero as the system size is increased. No exact results are known for the multichannel quantum wires from $\mathrm{BdG}$ symmetry classes in the absence of spin-rotation invariance (classes D and DIII; see, however, Refs. 34-36 for asymptotic results in one and two dimensions). The general scheme that we present here fills this gap, and provides a unified framework for all ten symmetry classes. ${ }^{37}$

\section{MAPPING TO QUANTUM WIRES WITH ABSORPTION}

Our construction of a scaling equation describing the DOS makes use of a mapping to a quantum wire with absorption. Absorption is described by the addition of a spatially uniform imaginary potential $i \omega, \omega>0$, to the Hamiltonian. In the presence of absorption, a scaling equation for the reflection eigenvalues $R_{j}, j=1, \ldots, N$, of $r(i \omega)^{\dagger} r(i \omega)$ can be derived in the same way as the scaling equation (2) in the absence of absorption, ${ }^{38,39}$ noting that upon the addition of a thin slice of length $\delta L$ to a disordered wire of length $L$, the reflection eigenvalues $R_{j}$ change by a small amount $\delta R_{j}$. The change $\delta R_{j}$ comes from two statistically independent contributions: (1) disorder scattering in the added slice of width $\delta L$, and (2) absorption in the same slice. These contributions are statistically independent, since to linear order in $\delta L$ one can neglect processes that involve both disorder scattering and absorption. The second process gives rise to a change

$$
R_{j} \rightarrow e^{-4 \omega \delta L / v_{F}} R_{j}, \quad j=1, \ldots, N,
$$


independent of the disorder. The first process depends on the disorder type and symmetries, and is already present in the scaling equation (2). Combination of the two processes then results in the replacement of Eq. (2) by

$$
\frac{\partial P_{i \omega}}{\partial L}=\sum_{j=1}^{N} \frac{\partial}{\partial x_{j}}\left(\frac{\omega}{v_{F}} \sinh 2 x_{j}+\frac{1}{2 \gamma l} J \frac{\partial}{\partial x_{j}} J^{-1}\right) P_{i \omega},
$$

where we used the parametrization (1) for the $R_{j}$, and $P_{i \omega}\left(x_{1}, \ldots, x_{N} ; L\right)$ represents the joint distribution of the reflection eigenvalue in the presence of the imaginary potential $i \omega$. Equation (6) was derived in Refs. 38 and 39 for the standard symmetry classes. The initial condition at $L=0$ for $P_{i \omega}\left(x_{1}, \ldots, x_{N} ; L\right)$ still needs to be specified. For our purposes, it is advantageous to set $R_{j}=1, j=1, \ldots, N$, at $L$ $=0$, corresponding to a wire that is closed on one end, see Fig. 1(b).

The scaling equation (6) has the stationary (independent of $L$ ) solution,

$$
P_{i \omega ; \mathrm{st}}\left(x_{1}, \ldots, x_{N}\right)=\frac{1}{Z(a)}|J| \prod_{j=1}^{N} e^{-a \cosh 2 x_{j}},
$$

where $a=\gamma l \omega / v_{F}$ and the normalization constant $Z(a)$ ensures that the stationary solution $P_{i \omega \text {; st }}$ is normalized to one. This solution represents the joint probability density of the reflection coefficients for the absorbing medium. We will demonstrate below that the DOS in an infinitely long disordered wire without absorption can be computed as a logarithmic derivative of the normalization constant or "partition function" $Z(a)$ with respect to the dimensionless imaginary energy $a$.

The main idea of our method is to make the analytical continuation $i \omega \rightarrow \varepsilon$. With this analytical continuation, the single-energy product $r(i \omega)^{\dagger} r(i \omega)$ of reflection matrices in the presence of absorption maps to the two-energy product $r(-\varepsilon)^{\dagger} r(\varepsilon)$ without absorption. ${ }^{40}$ The latter product is a unitary matrix since the reflection matrix in the geometry of Fig. 1(b) is unitary by construction. The $N$-independent eigenvalues of $r(-\varepsilon)^{\dagger} r(\varepsilon)$ can thus be written as $\exp \left(2 i \phi_{j}\right)$, $j=1, \ldots, N$. When the length $L$ of the disordered wire is increased, the $\phi_{j}$ 's perform a Brownian motion, governed by a Fokker-Planck equation that is the analytical continuation of Eq. (6),

$$
\frac{\partial P_{\varepsilon}}{\partial L}=\sum_{j=1}^{N} \frac{\partial}{\partial \phi_{j}}\left(-\frac{2 \varepsilon}{v_{F}}+\frac{2}{\gamma l} \sin ^{2} \phi_{j} J \frac{\partial}{\partial \phi_{j}} J^{-1}\right) P_{\varepsilon},
$$

where

$$
J=\prod_{j=1}^{N} \frac{1}{\sin ^{\gamma} \phi_{j}} \prod_{k<j} \sin ^{m} o\left(\phi_{j}-\phi_{k}\right)
$$

for the standard and BdG classes, and

$$
J=\prod_{j=1}^{N} \frac{1}{\sin ^{\gamma} \phi_{j}} \prod_{k<j} \sin ^{m_{o}}\left[\left(\phi_{j}-\phi_{k}\right) / 2\right]
$$

for the chiral classes, where $\gamma$ is defined in Eqs. (3) and (4), respectively. This equation was derived by different methods in Ref. 31 for the chiral classes and in Ref. 41 for the standard classes. The scaling equation (8) can be considered either on the full real axis $-\infty<\phi_{j}<\infty$ or on the interval $-\pi \leqslant \phi_{j}<\pi$. Only in the latter case has Eq. (8) a welldefined stationary solution $P_{\varepsilon ; \text { st }}\left(\left\{\phi_{j}\right\}\right)$ describing the distribution $P_{\varepsilon}\left(\left\{\phi_{j}\right\} ; L\right)$ in the limit $L \rightarrow \infty$. However, this solution is not of the simple form (7). ${ }^{42}$ In fact, no representation of $P_{\varepsilon ; \text { st }}$ in terms of elementary functions could be found other than for the case $N=1$, where explicit integration of Eq. (8) is possible. ${ }^{43}$

The DOS is calculated via the "node-counting theorem," which states that, for the half-open quantum wire of Fig. 1(b), the mean number of states $\mathcal{N}(\varepsilon)$ in the energy interval $(-\varepsilon, \varepsilon)$ and per unit length is proportional to the sum of the phase derivatives $\partial \phi_{j} / \partial L .^{44,45}$ For technical reasons, we replace $\partial \phi_{j} / \partial L$ by the derivative of the counting function $-\operatorname{Im} \ln \sin \left(\phi_{j}+i 0\right)$, which has the same average slope as $\phi_{j}$. We thus find

$$
\mathcal{N}(\varepsilon) \equiv \int_{-\varepsilon}^{\varepsilon} d \varepsilon^{\prime} \nu\left(\varepsilon^{\prime}\right)=-\frac{d}{\pi} \sum_{j=1}^{N} \frac{\partial}{\partial L}\left\langle\operatorname{Im} \ln \sin \left(\phi_{j}+i 0\right)\right\rangle_{\varepsilon, L} .
$$

Note that the positive infinitesimal $i 0$ in the argument of $\sin \left(\phi_{j}+i 0\right)$, which is needed to select the correct branch of the logarithm, is compatible with the analytical continuation $i \omega \rightarrow \varepsilon$ used to obtain the scaling equation (8). In Eq. (9) the brackets $\langle\ldots\rangle_{\varepsilon, L}$ denote an average with the probability density $P_{\varepsilon}$ that solves Eq. (8) on the full real axis $-\infty<\phi_{j}$ $<\infty$ at length $L$ of the quantum wire. Integrating by parts, with the help of Eq. (8), we find

$$
\begin{aligned}
\mathcal{N}(\varepsilon)= & -\frac{d}{\pi} \sum_{j=1}^{N} \int d \phi_{1} \cdots d \phi_{N} \operatorname{Im}\left[\cot \left(\phi_{j}+i 0\right)\right] \\
& \times\left(\frac{2 \varepsilon}{v_{F}}-\frac{2}{\gamma l} \sin ^{2} \phi_{j} J \frac{\partial}{\partial \phi_{j}} J^{-1}\right) P_{\varepsilon} \\
= & -\frac{2 d \varepsilon}{\pi v_{F}} \sum_{j=1}^{N}\left\langle\operatorname{Im} \cot \left(\phi_{j}+i 0\right)\right\rangle_{\varepsilon, L} .
\end{aligned}
$$

In the thermodynamic limit $L \rightarrow \infty$, the disorder average $\langle\cdots\rangle_{\varepsilon, L}$ can be performed with the stationary distribution $P_{\varepsilon ; \text { st }}$ for the interval $-\pi \leqslant \phi_{j}<\pi$. Denoting the average with $P_{\varepsilon, \mathrm{st}}$ by $\langle\cdots\rangle_{\varepsilon}$, the DOS per unit length is thus given by

$$
\nu(\varepsilon)=\frac{1}{2} \frac{\partial \mathcal{N}}{\partial \varepsilon}=-\frac{d}{\pi v_{F}} \sum_{j=1}^{N} \frac{\partial}{\partial \varepsilon} \varepsilon\left\langle\operatorname{Im} \cot \left(\phi_{j}+i 0\right)\right\rangle_{\varepsilon},
$$

where we have used the relation

$$
\nu(\varepsilon)=\nu(-\varepsilon)
$$

that holds for each disorder configuration in the chiral and BdG classes. In the standard classes, Eq. (12) only holds in the thermodynamic limit and for sufficiently small $\varepsilon$ (smaller 
than the energy scale $v_{F} / l$, where nonuniversal effects appear and the validity of the scaling equations breaks down).

Instead of performing the disorder average with the stationary solution $P_{\varepsilon ; \text { st }}$ of Eq. (8), we rely on the solution $P_{i \omega ; s t}$ for the stationary distribution of the reflection eigenvalues in the presence of the imaginary potential $i \omega$, see Eq. (7). From the relation $\tanh ^{2} x_{j}=e^{2 i \phi_{j}}$ we first observe that

$$
\operatorname{Im}\left\langle\cot \left(\phi_{j}+i 0\right)\right\rangle_{\varepsilon}=-\left.\operatorname{Re}\left\langle\cosh 2 x_{j}\right\rangle_{i \omega}\right|_{\omega \rightarrow-i \varepsilon},
$$

where $\langle\cdots\rangle_{i \omega}$ stands for averaging with the stationary distribution $P_{i \omega ; \mathrm{st}}$. Second, we note that the average $-\Sigma_{m}\left\langle\cosh 2 x_{m}\right\rangle_{i \omega}$ is given by the logarithmic derivative of the partition function $Z(a)$, thus obtaining the DOS

$$
\nu(\varepsilon)=-\left.\frac{d}{\pi v_{F}} \operatorname{Re} \frac{\partial}{\partial a}\left[a \frac{\partial}{\partial a} \ln Z(a)\right]\right|_{a \rightarrow-i \gamma / \varepsilon / v_{F}} .
$$

This relation between the DOS and the partition function $Z(a)$ for each of the Cartan symmetry classes is the key result of this paper.

\section{CALCULATION OF THE DENSITY OF STATES}

As we have seen in the preceding section, the computation of the DOS in the thermodynamic limit reduces to the computation of the "partition function"

$$
Z(a)=\int d x_{1} \cdots d x_{N}\left|J\left(\left\{x_{j}\right\}\right)\right| e^{-a \sum_{j=1}^{N} \cosh 2 x_{j} .}
$$

For the standard and BdG classes, the integration over the coordinates $x_{j}$ is restricted to the positive real axis, $0<x_{j}$ $<\infty, j=1, \ldots, N$, while for the chiral classes, the integration extends over the entire real axis.

For all ten symmetry classes, a change of variables $x_{j}$ $\rightarrow \lambda_{j}$ can be made after which $Z(a)$ can be written in the form

$$
Z \propto \int_{\mathcal{L}} d \lambda_{1} \cdots d \lambda_{N} \prod_{j=1}^{N} w\left(\lambda_{j}\right) \prod_{k<j}\left|\lambda_{j}-\lambda_{k}\right|^{m_{o}} .
$$

The relation between the $\lambda_{j}$ and the $x_{j}$, the weight function $w(\lambda)$, and the integration interval $\mathcal{L}$ depend on the symmetry class and will be specified below.

A general method for calculation of integrals of the type (16) is described in Chaps. 5 and 6 of Mehta's book on random matrix theory. ${ }^{4}$ Here we mention the results from Ref. 4, up to a proportionality constant that does not depend on $a$. For $m_{o}=2$ the result of integration in Eq. (16) is proportional to the determinant

$$
\begin{gathered}
Z \propto \operatorname{Det}\left[f_{m n}\right]_{m, n=1}^{N}, \\
f_{m n}=\int_{\mathcal{L}} d \lambda w(\lambda) p_{m}(\lambda) p_{n}(\lambda),
\end{gathered}
$$

where the polynomials $\left\{p_{m}(\lambda)\right\}$ form a linear independent set of polynomials up to degree $N-1$. For $m_{o}=1$ and even $N$ we have ${ }^{4}$

$$
\begin{gathered}
Z \propto \sqrt{\operatorname{Det}\left[f_{m n}\right]_{m, n=1}^{N}}, \\
f_{m n}=\int_{\mathcal{L}} d \lambda d \lambda^{\prime} \epsilon\left(\lambda-\lambda^{\prime}\right) w(\lambda) w\left(\lambda^{\prime}\right) p_{m}(\lambda) p_{n}\left(\lambda^{\prime}\right),
\end{gathered}
$$

where the sign function $\epsilon(\lambda)$ equals 1 for positive $\lambda$ and -1 for negative $\lambda$. For odd $N$, the matrix $f$ must be supplemented by an additional row and column

$$
\begin{gathered}
Z \propto \sqrt{\operatorname{Det}\left[f_{m n}\right]_{m, n=1}^{N+1}}, \\
f_{n, N+1}=-f_{N+1, n}=\int_{\mathcal{L}} d \lambda w(\lambda) p_{n}(\lambda) .
\end{gathered}
$$

For $m_{o}=4$, the result is

$$
\begin{gathered}
Z \propto \sqrt{\operatorname{Det}\left[f_{m n}\right]_{m, n}^{2 N}}, \\
f_{m n}=\int_{\mathcal{L}} d \lambda w(\lambda)\left[p_{m}(\lambda) p_{n}^{\prime}(\lambda)-p_{n}(\lambda) p_{m}^{\prime}(\lambda)\right],
\end{gathered}
$$

where the prime stands for the derivative with respect to $\lambda$ and $\left\{p_{n}(\lambda)\right\}$ form a linear independent set of polynomials up to degree $2 N-1$.

\section{A. Standard classes}

For the standard universality classes, the partition function $Z(a)$ takes the form of the normalization integral of the Laguerre ensemble of random matrix theory ${ }^{38}$ if we set $\lambda$ $=\sinh ^{2} x$. In this case, the integration range $\mathcal{L}=(0, \infty)$ and the weight function $w(\lambda)$ is given by $\exp (-2 a \lambda-a)$. Integrating over the $\lambda_{j}$, one finds that the partition function $Z(a)$ has a particularly simple dependence on $a$,

$$
Z(a) \propto e^{-a N} a^{-\gamma N / 2} .
$$

Using Eq. (14), one quickly verifies that this corresponds to a constant DOS,

$$
\nu(\varepsilon)=\nu_{0} \equiv \frac{N d}{\pi v_{F}} .
$$

(The DOS can acquire a nonuniversal energy dependence on the scales $\varepsilon$ much larger then the inverse scattering time $v_{F} / l$, where the applicability of the scaling equation breaks down.)

As a matter of fact, for large $a$, one obtains the result (21) for all ten symmetry classes, as one verifies by substitution $x_{j} \rightarrow a^{-1 / 2} y_{j}$ in Eq. (15) and subsequent expansion for large $a$. This confirms that the DOS $\nu(\varepsilon)$ approaches the standard value $\nu_{0}$ for energies far away from zero for all symmetry classes.

\section{B. Chiral classes}

For the chiral classes, we arrive at the standard form (16) taking $\lambda=\exp (2 x), \mathcal{L}=(0, \infty)$, and

$$
w(\lambda)=\lambda^{-\gamma / 2} \exp \left[-\frac{1}{2} a\left(\lambda+\lambda^{-1}\right)\right] .
$$


When TR is broken, say by a magnetic field $\left(m_{o}=2\right.$; class AIII), we choose in Eq. (17) the polynomials $p_{n}(\lambda)$ $=\lambda^{n-1}$ so that

$$
Z(a) \propto \operatorname{Det}\left[K_{|m-n|}(a)\right]_{m, n=1}^{N},
$$

where $K_{j}(a)$ is the modified Bessel function of integer order $j$. For small values of the dimensionless imaginary energy $a$ one can substitute $K_{0}(a) \rightarrow-\ln a$ and $K_{m}(a)$ $\rightarrow 2^{m-1} a^{-m}(m-1) !, m=1,2, \ldots$, to obtain the leading behavior of $Z$ (to logarithmic accuracy). For odd $N$ the determinant in Eq. (24) is expanded according to

$$
Z(a) \propto a^{-\left(N^{2}-1\right) / 2}[\ln a+\mathcal{O}(1)] .
$$

This gives the asymptotic DOS

$$
\nu(\varepsilon)=\frac{\pi \nu_{0}}{\left|\varepsilon \tau \ln ^{3}(\varepsilon \tau)\right|}, \quad 0<\varepsilon \tau \ll 1,
$$

where we introduced the time scale

$$
\tau=N \gamma l / v_{F} .
$$

It is the time needed to diffuse through a wire of length $\sim N l$, or, equivalently, the mean DOS in a segment of the wire of length $N l$. (The length scale $N l$ characterizes the crossover between the regimes of diffusive dynamics and of localized or critical dynamics.) For even $N$ the determinant in Eq. (24) is expanded according to

$$
Z(a) \propto a^{-N^{2} / 2}\left[1-\frac{1}{4} N^{2} a^{2} \ln ^{2} a+\mathcal{O}\left(a^{2} \ln a\right)\right] .
$$

This gives rise to a different asymptotic DOS,

$$
\nu(\varepsilon)=\pi \nu_{0}|\varepsilon \tau \ln (\varepsilon \tau)|, \quad 0<\varepsilon \tau \ll 1 .
$$

Observe that the asymptotic results (26a) and (28) do not depend explicitly on $N$ when the energy is measured in units of $1 / \tau$. The leading dependence on energy in Eqs. (26a) and (28) has been derived previously in Ref. 31 in an approximation that leaves the prefactor $\pi \nu_{0}$ unspecified.

When TR symmetry is present but SR symmetry is broken by spin-orbit coupling $\left(m_{o}=4\right.$, class CII), we choose in Eq. (20) the polynomials $p_{2 n-1}(\lambda)=\lambda^{2 N-n}+\lambda^{n-1}$ and $p_{2 n}(\lambda)=\lambda^{2 N-n}-\lambda^{n-1}$. With such a choice the square root of the determinant of a $2 N \times 2 N$ matrix in Eq. (20) reduces to the determinant of a $N \times N$ matrix, from which we obtain

$$
\begin{gathered}
Z(a) \propto \operatorname{Det}\left[f_{n m}\right]_{n, m=1}^{N}, \\
f_{n m}=(2 N-n-m+1) K_{|n-m|}(a)+(n-m) K_{2 N-n-m+1}(a) .
\end{gathered}
$$

For odd $N$, the leading term in the asymptotic expansion of $Z$ ( $a$ ) for small $a$ is of the form (25), hence the small- $\varepsilon$ asymptote of the DOS is given by Eq. (26a), just like in the case of broken time-reversal symmetry. For even $N$ the determinant in Eq. (29) is expanded as

$$
Z(a) \propto a^{-N^{2}}\left[1+c_{1} a^{2}+c_{2} a^{4} \ln ^{2} a+\mathcal{O}\left(a^{4} \ln a\right)\right],
$$

where $c_{2}=\frac{1}{48} N^{2}(N+1)^{2}$ and $c_{1}$ is another $N$-dependent coefficient, which does not affect the asymptote of the DOS. We thus obtain

$$
\nu(\varepsilon)=\frac{\pi}{3} \nu_{0}\left(1+\frac{1}{N}\right)^{2}\left|(\varepsilon \tau)^{3} \ln (\varepsilon \tau)\right|, \quad 0<\varepsilon \tau \ll 1
$$

In the presence of both TR and SR symmetry $\left(m_{o}=1\right.$, class BDI) and with the choice of the polynomials $p_{n}(\lambda)=\lambda^{n-1}$, $n=1, \ldots, N$, we obtain for even $N$

$$
\begin{aligned}
& Z(a) \propto \sqrt{\operatorname{Det}\left[f_{m n}\right]_{m, n=1}^{N}} \\
& f_{m n}=\int_{0}^{\infty} d x \int_{x}^{\infty} d y(x y)^{-(1 / 2)(N+1)} e^{-(1 / 2) a\left(x+y+x^{-1}+y^{-1}\right)} \\
& \times\left(x^{n-1} y^{m-1}-x^{m-1} y^{n-1}\right)
\end{aligned}
$$

For odd $N$, the antisymmetric matrix $f$ has to be supplemented by an additional row and column with

$$
\begin{aligned}
f_{n, N+1} & =\int_{0}^{\infty} d x x^{-(1 / 2)(N+1)} e^{-(1 / 2) a\left(x+x^{-1}\right)} x^{n-1} \\
& =2 K_{|(N+1) / 2-n|}(a) .
\end{aligned}
$$

The analytical continuation $a \rightarrow-i \gamma l \varepsilon / v_{F}=-i \tau \epsilon / N$ can be done directly in the integrals (34), (35) if accompanied by an appropriate shift of the integration contour of the variables $x$ and $y$ in the complex plane. After that, numerical evaluation of the partition function (33) for complex $a$ and its derivatives, and hence of the DOS $\nu(\epsilon)$, is straightforward. ${ }^{46}$ The small- $a$ asymptote of the partition function $Z(a)$ is given by Eq. (25) for odd $N$ and by

$$
Z(a)=a^{-(N / 2)^{2}}[1+c a \ln a+\mathcal{O}(a)]
$$

for even $N$, where $c=\pi[(N / 2-1) ! ! /(N / 2-2) ! !]^{2}$ if $N$ $=4 n$ and $c=4 \pi^{-1}[(N / 2-1) ! ! /(N / 2-2) ! !]^{2}$ if $N=4 n$ $-2, n=1,2, \ldots$. As a result, for small energies, the DOS has the form (26a) for odd $N$, while

$$
\nu(\varepsilon)=\frac{\nu_{0} c}{N}|\ln (\varepsilon \tau)|, \quad 0<\varepsilon \tau \ll 1
$$

for even $N$, where the coefficient $c$ is given below Eq. (36). In the limit $N \gg 1$, this asymptote simplifies to $\nu(\varepsilon)$ $=\nu_{0}|\ln (\varepsilon \tau)|, 0<\varepsilon \tau \ll 1$.

The DOS is plotted for the three chiral classes and for various values of $N$ in Fig. 2. The parity effect and the dependence on symmetry class is clearly seen.

\section{BdG universality classes}

We now apply Eqs. (17)-(20) to the BdG universality classes. We choose $\lambda=\cosh 2 x, \mathcal{L}=(1, \infty)$, and

$$
w(\lambda)=\exp (-a \lambda)\left(\lambda^{2}-1\right)^{\left(m_{l}-1\right) / 2},
$$

to arrive at the standard form (16). In all calculations below we choose the polynomials $p_{n}(\lambda)=\lambda^{n-1}$ to compute $Z(a)$. 

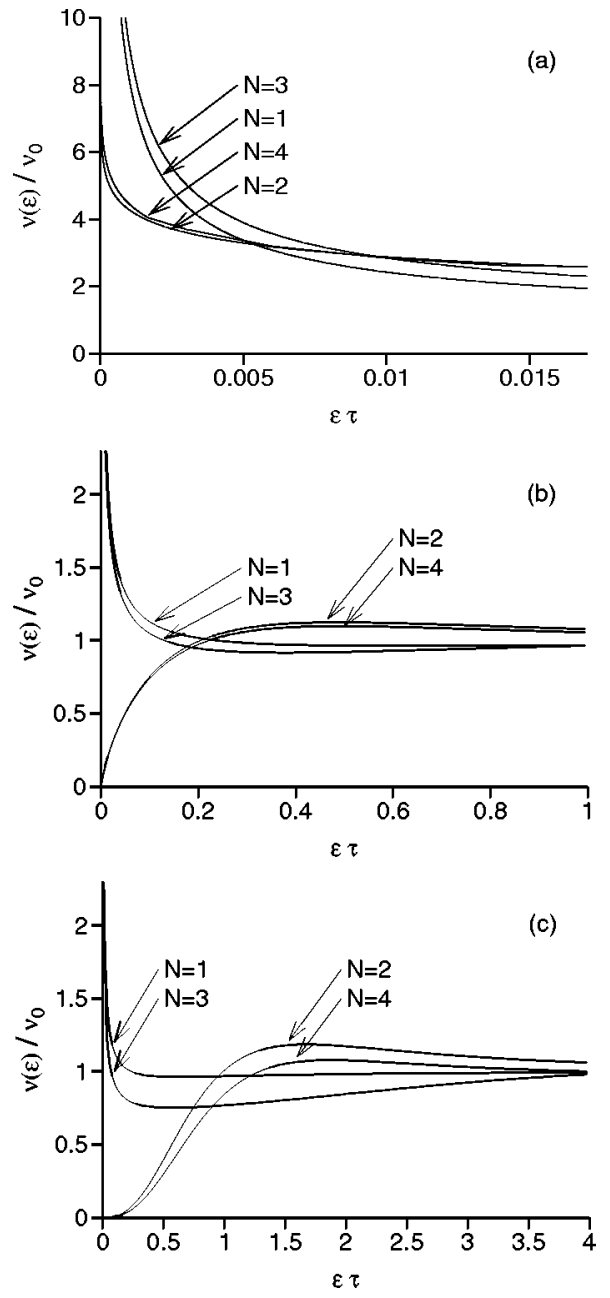

FIG. 2. Density of states for the chiral classes. The energy is measured in units of $1 / \tau=v_{F} /(N \gamma l)$; the DOS is measured in units of $\nu_{0}=N d / \pi v_{F}$. (a) is for the case of preserved time-reversal symmetry and spin-rotation invariance (Hamiltonian of class BDI in Cartan's classification), (b) is for the case of broken time-reversal symmetry (class AIII), and (c) is for the case of preserved timereversal symmetry and broken spin-rotation invariance (class CII). The density of states is shown for $N=1,2,3$, and 4; the values of $N$ are indicated in the figure.

When both TR and SR symmetries are present (class CI), say as is the case with a dirty $d$-wave superconductor, we use Eq. (17) and find

$$
Z(a) \propto \operatorname{Det}\left[\left(-\frac{d}{d a}\right)^{m+n-2} a^{-1} K_{1}(a)\right]_{m, n=1}^{N} .
$$

With the help of the asymptotic limit $a \rightarrow 0$ of the modified Bessel function $K_{1}$, we obtain

$$
Z(a) \propto a^{-N(N+1)}\left[1+\frac{1}{4} N(N+1) a^{2} \ln a+\mathcal{O}\left(a^{2}\right)\right] .
$$

Substituting $a \rightarrow-i \tau \varepsilon / N$ and using Eq. (14) one verifies that the DOS vanishes linearly with energy as $\varepsilon \rightarrow 0$

$$
\nu(\varepsilon)=\frac{\pi}{2} \nu_{0}\left(1+\frac{1}{N}\right)|\varepsilon \tau|, \quad 0<\varepsilon \tau \ll 1 .
$$

When SR symmetry is present but not TR (class C), we find

$$
\begin{gathered}
Z(a) \propto \sqrt{\operatorname{Det}\left[f_{m n}\right]_{m, n=1}^{2 N}}, \\
f_{m n}=(n-m)\left(-\frac{d}{d a}\right)^{n+m-3} \frac{1+a}{a^{3}} e^{-a} .
\end{gathered}
$$

In the limit $a \rightarrow 0$, we obtain

$$
Z(a) \propto a^{-N(2 N+1)}\left[1+c_{1} a^{2}+c_{2} a^{3}+\mathcal{O}\left(a^{4}\right)\right],
$$

where $c_{2}=\frac{1}{18} N(N+1)(2 N+1)$ and $c_{1}$ is a numerical coefficient which is not important for the small energy asymptote of the DOS. From Eq. (43), one verifies that the DOS vanishes quadratically with energy as $\varepsilon \rightarrow 0$,

$$
\nu(\varepsilon)=\nu_{0}\left(1+\frac{1}{N}\right)\left(1+\frac{1}{2 N}\right)(\varepsilon \tau)^{2}, \quad 0<\varepsilon \tau \ll 1 .
$$

The linear and quadratic energy dependencies of the DOS in the thermodynamic limit and for small energies in the classes $\mathrm{CI}$ and $\mathrm{C}$, respectively, have been found previously by Senthil and co-workers ${ }^{18,33}$ The same suppression of the DOS appears in the random matrix theory for finite-size systems of classes CI and $\mathrm{C}^{6}{ }^{6}$ provided the size of the system is set equal to a localization volume (corresponding to a segment of length $N l$ of the quantum wire).

When TR symmetry is present but not SR (class DIII), we find from Eq. (17)

$$
Z(a) \propto \operatorname{Det}\left[\left(-\frac{d}{d a}\right)^{m+n-2} K_{0}(a)\right]_{m, n=1}^{N} .
$$

Note that $N$ is even for the class DIII. In the limit $a \rightarrow 0$, using the asymptotic expansion of the modified Bessel function $K_{0}$, we obtain $Z(a) \propto a^{-N(N-1)}[\ln a+\mathcal{O}(1)]$, i.e., corresponding to a DOS of the form (26a). For class DIII, the case $N=2$ is special: The Lie algebra $\operatorname{so}(4, \mathrm{C})$ generated by the transfer matrices of a quantum wire in class DIII with $N$ $=2$ (see Ref. 30) is not irreducible, $\operatorname{so}(4, \mathrm{C}) \approx \operatorname{sl}(2, \mathrm{C})$ $\times s l(2, \mathrm{C})$ (see Ref. 1). As a result, in the case DIII with $N$ $=2$ the scaling of the parameters $x_{j}$ and of the eigenphases $\phi_{j}$ is described by two mean free paths that need not be equal; one for each copy of the noncompact Lie algebra $\operatorname{sl}(2, \mathrm{C})$. The scaling equations (2), (6), and (8), in which only one mean free path appears, correspond to the special case where these two mean free paths are equal, which requires fine tuning of the disorder. Only in that case the DOS singularity (26a) is found. In Refs. 34 and 35 it is shown that the general case of different mean free paths has a power law dependence of the DOS for $\varepsilon \rightarrow 0$ with a nonuniversal exponent.

Finally, when neither SR nor TR symmetries are present (class D), it is enough to use only Eq. (18) because the number of eigenvalues $N$ is a multiple of four in this case. We then obtain

$$
Z(a) \propto \sqrt{\operatorname{Det}\left[f_{m n}\right]_{m, n=1}^{N}},
$$



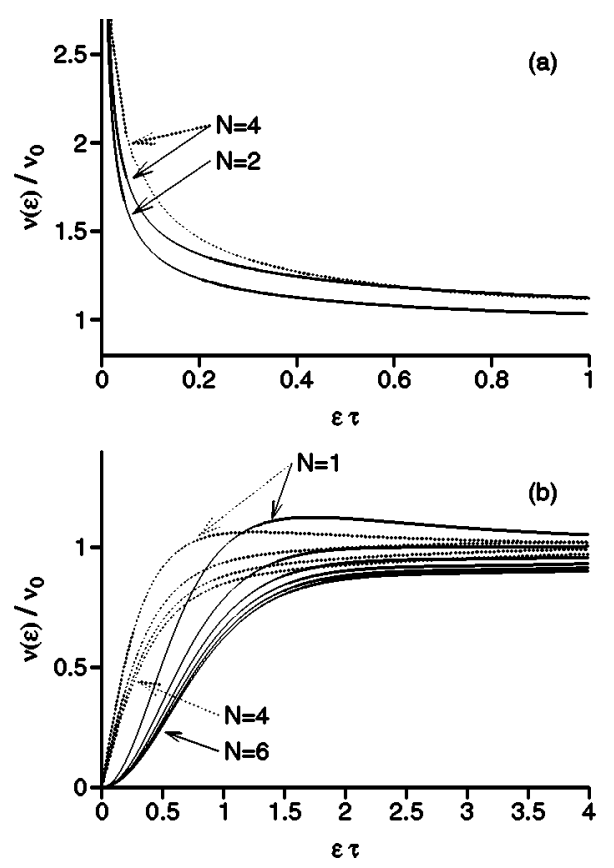

TABLE II. Asymptotes of the DOS $\nu(\varepsilon)$ for small energies 0 $<\varepsilon \tau \ll 1$ and for $N \gg 1$, where $\tau=N \gamma l / v_{F}, \gamma$ is given in Eqs. (3b) and (4b), $l$ is the mean free path, and $v_{F}$ the Fermi velocity. (Expressions for the DOS asymptotes for finite $N$ can be found in the text.)

\begin{tabular}{|c|c|c|c|}
\hline Class & TR & SR & $\nu(\varepsilon)$ for $0<\varepsilon \tau \ll 1$ \\
\hline \multirow{3}{*}{ Chiral, $N$ even } & Yes & Yes & $\nu_{0}|\ln (\varepsilon \tau)|$ \\
\hline & No & & $\pi \nu_{0}|\varepsilon \tau \ln (\varepsilon \tau)|$ \\
\hline & Yes & No & $\left(\pi \nu_{0} / 3\right)\left|(\varepsilon \tau)^{3} \ln (\varepsilon \tau)\right|$ \\
\hline \multirow[t]{2}{*}{ Chiral, $N$ odd } & & & $\pi \nu_{0} /\left|\varepsilon \tau \ln ^{3}(\varepsilon \tau)\right|$ \\
\hline & Yes & Yes & $\left(\pi \nu_{0} / 2\right)|\varepsilon \tau|$ \\
\hline \multirow[t]{3}{*}{ BdG } & No & Yes & $\nu_{0}|\varepsilon \tau|^{2}$ \\
\hline & Yes & No & $\pi \nu_{0} /\left|\varepsilon \tau \ln ^{3}(\varepsilon \tau)\right|$ \\
\hline & No & No & $\pi \nu_{0} /\left|\varepsilon \tau \ln ^{3}(\varepsilon \tau)\right|$ \\
\hline
\end{tabular}

wire, for all ten (pure) Cartan symmetry classes. ${ }^{37}$ While it was well known that the dependence of the conductance on the length $L$ of the quantum wire could be inferred from the theory of diffusion on symmetric spaces through the Dorokhov-Mello-Pereyra-Kumar (DMPK) equation and its generalizations, ${ }^{3,22,23}$ we have shown that the same geometrical framework underlies the dependence of the DOS on the energy $\varepsilon$ (measured with respect to the band center or Fermi energy $\varepsilon=0$ ). Our unified picture consists of a general scheme that permits the construction of scaling equations for the eigenvalues $e^{2 i \phi_{j}}$ of the matrix product $r^{\dagger}(-\varepsilon) r(\varepsilon)$ of reflection matrices $r$ at different energies for a disordered quantum wire and for all ten pure Cartan symmetry classes. [Here $j=1, \ldots, N$, where $N$ is the number of independent eigenvalues of $r^{\dagger}(-\varepsilon) r(\varepsilon)$.] Using the analytical continuation $\varepsilon \rightarrow i \omega$, this scaling equation could be obtained from that for the reflection eigenvalues $R_{j}$ of the quantum wire in the presence of absorption, which, in turn, can be obtained from the DMPK equation. Using the "node-counting theorem" that relates the $L$ dependence of the eigenphases $\phi_{j}$ and the DOS ${ }^{44,45}$ we were thus able to find the DOS in the thermodynamic limit $L \rightarrow \infty$ from the stationary solution of the scaling equation.

Relatively little attention has been paid to the behavior of the density of states (DOS) in the standard problem of Anderson localization for a system of infinite size, since this DOS, with the exception of its tails, is rather insensitive to disorder. This is not true in the presence of symmetries for which one energy in the spectrum plays a special role, as is the case for the chiral and Bogoliubov-de Gennes (BdG) universality classes, where a sublattice symmetry or the presence of a superconducting order parameter singles out the band center or the Fermi energy $\varepsilon=0$, respectively. In those cases, the DOS is singular around zero energy, the energy scale for the singularity being $\hbar / \tau$, where, for the case of a long quantum wire we consider in this paper, $\tau \sim N^{2} l / v_{F}$ is the time for diffusion through a segment of the wire of length $\mathrm{Nl}$. The precise form of the singularity depends on the symmetry class and, for the chiral classes, on the parity of $N$. The results are summarized in Table II for the limit of a multichannel quantum wire, $N \gg 1$. It is in this limit that a univer-

In this paper, we have presented a unified picture for both transport and the density of states (DOS) in a long quantum 
sal dependence of the DOS on the symmetry class is expected.

One might be tempted to argue that, for those classes where the quasiparticle states are localized at and near zero energy, the singular behavior of the DOS in the thermodynamic limit is controlled by the (zero-dimensional) random matrix theory for an effective finite-size system with the linear size of the localization length $\xi=N l$. Whereas this argument predicts the correct energy scale $\hbar / \tau$ for the singularity, it fails to reproduce the extra logarithm in the functional form of the singularity for the chiral classes, see Table II. The origin of the logarithm in the DOS can be found in the presence of two competing localized modes in that case, that each have the same localization length, see Refs. 29 and 31. As was shown in Ref. 31, the presence of a small amount of dimerization of the hopping amplitudes lifts the degeneracy of the localization lengths and removes the logarithm from the functional form of the DOS singularity. On the other hand, for the BdG classes C and CI, there is only one localized mode,$^{30}$ which explains the absence of the extra loga- rithm in the functional form of the DOS in those classes.

Note added. Upon completion of this manuscript, we learned of a related paper by Motrunich et al. (Ref. 34) on single-channel quantum wires with BdG symmetry and broken spin-rotation invariance, where the same conclusion was reached with respect as to the DOS singularity for the pure Cartan symmetry classes D and DIII.

\section{ACKNOWLEDGMENTS}

We thank C. W. J. Beenakker, K. Damle, and I. A. Gruzberg for discussions. This work was supported by the Dutch Science Foundation NWO/FOM and by INTAS Grant No. 97-1342 (M.T.), the Sloan Foundation (P.W.B.), and by the Grant-in-Aid for Scientific Research on Priority Areas (A) from the Ministry of Education, Science, Sports and Culture of Japan No. 12046238 (A.F.). P.W.B. gratefully acknowledges the hospitality of the Instituut Lorentz of Leiden University where part of this work was done.

${ }^{1}$ S. Helgason, Differential Geometry, Lie Groups, and Symmetric Spaces (Academic Press, San Diego, 1978).

${ }^{2}$ M. Zirnbauer, J. Math. Phys. 37, 4986 (1996).

${ }^{3}$ M. Caselle, cond-mat/9610017 (unpublished).

${ }^{4}$ M.L. Mehta, Random Matrices and the Statistical Theory of Energy Levels (Academic Press, New York, 1967).

${ }^{5}$ J. Verbaarschot and I. Zahed, Phys. Rev. Lett. 70, 3852 (1993); T. Nagao and S. Slevin, J. Math. Phys. 34, 2075, 2317 (1993); S. Hikami and A. Zee, Nucl. Phys. B 408, 415 (1993).

${ }^{6}$ A. Altland and M.R. Zirnbauer, Phys. Rev. B 55, 1142 (1997).

${ }^{7}$ In the standard and chiral classes, the symmetry classes with and without spin-rotation invariance coincide once time-reversal symmetry is broken.

${ }^{8}$ P.W. Anderson, Phys. Rev. 109, 1492 (1958).

${ }^{9}$ R. Gade, Nucl. Phys. B 398, 499 (1993); R. Gade and F. Wegner, ibid. 360, 213 (1991).

${ }^{10}$ A. Furusaki, Phys. Rev. Lett. 82, 604 (1999); A. Altland and B.D. Simons, Nucl. Phys. B 562, 445 (1999), and references therein.

${ }^{11}$ C. Mudry, P.W. Brouwer, and A. Furusaki, Phys. Rev. B 59, 13221 (1999); 62, 8249 (2000).

${ }^{12}$ Y. Hatsugai, X.-G. Wen, and M. Kohmoto, Phys. Rev. B 56, 1061 (1997); T. Fukui, Nucl. Phys. B 562, 477 (1999); S. Guruswamy, A. LeClair, and A.W.W. Ludwig, ibid. 583, 475 (2000).

${ }^{13}$ A. Eilmes, R.A. Römer, and M. Schreiber, Eur. Phys. J. B 1, 29 (1998); M. Fabrizio and C. Castellani, Nucl. Phys. B 583, 542 (2000), and references therein.

${ }^{14}$ E.R. Smith, J. Phys. C 3, 1419 (1970); R. Shankar and G. Murthy, Phys. Rev. B 36, 536 (1987); D.S. Fisher, ibid. 50, 3799 (1994); 51, 6411 (1995).

${ }^{15}$ Depending on the type of disorder, unconventional superconductors are sometimes described by the chiral classes; see A. Altland, B.D. Simons, and M.R. Zirnbauer, cond-mat/0006362 (unpublished).

${ }^{16}$ M.A. Skvortsov and M.V. Feigel'man, Phys. Rev. Lett. 78, 2640 (1997).

${ }^{17}$ R. Bundschuh, C. Cassanello, D. Serban, and M.R. Zirnbauer, Nucl. Phys. B 532, 689 (1998).

${ }^{18}$ T. Senthil, M.P.A. Fisher, L. Balents, and C. Nayak, Phys. Rev. Lett. 81, 4704 (1998).

${ }^{19}$ The BdG symmtetry classes are only appropriate when the disorder is weak. In the opposite case of unitary impurity scattering, different behavior is found. See C. Pépin and P.A. Lee, Phys. Rev. Lett. 81, 2779 (1998); Phys. Rev. B 63, 054502 (2001); W.A. Atkinson, P.J. Hirschfeld, A.H. MacDonald, and K. Ziegler, Phys. Rev. Lett. 85, 3922 (2000); 85, 3926 (2000); J.-X. Zhu, D.N. Sheng, and C.S. Ting, ibid. 85, 4944 (2000); C. Chamon and C. Mudry, Phys. Rev. B 63, 100503(R) (2001).

${ }^{20}$ S. Cho and M.P.A. Fisher, Phys. Rev. B 55, 1025 (1997); N. Read and D. Green, ibid. 61, 10267 (2000); M. Bocquet, D. Serban, and M.R. Zirnbauer, Nucl. Phys. B 578, 628 (2000); I.A. Gruzberg, N. Read, and A.W.W. Ludwig, Phys. Rev. B 63, 104422 (2001); N. Read and A.W.W. Ludwig, Phys. Rev. B 63, 024404 (2001).

${ }^{21}$ V. Kagalovsky, B. Horovitz, Y. Avishai, and J.T. Chalker, Phys. Rev. Lett. 82, 3516 (1999); I.A. Gruzberg, N. Read, and A.W.W. Ludwig, ibid. 82, 4524 (1999); T. Senthil, J.B. Marston, and M.P.A. Fisher, Phys. Rev. B 60, 4245 (1999); J.T. Chalker, N. Read, V. Kagalovsky, B. Horovitz, Y. Avishai, and A.W.W. Ludwig, cond-mat/0009463 (unpublished).

${ }^{22}$ A. Hüffmann, J. Phys. A 23, 5733 (1990).

${ }^{23}$ C.W.J. Beenakker, Rev. Mod. Phys. 69, 731 (1997).

${ }^{24}$ The scaling equation is not generic for Cartan's class DIII if $N$ $=2$; see the discussion following Eq. (45).

${ }^{25}$ The multiplicity $m_{o}$ is known as the Dyson symmetry parameter $\beta$ for the standard ensembles from random matrix theory, see Ref. 4.

${ }^{26}$ P.W. Brouwer, C. Mudry, and A. Furusaki, Nucl. Phys. B 565[FS], 653 (2000). 
${ }^{27}$ O.N. Dorokhov, Pis'ma Zh. Éksp. Teor. Fiz. 36, 259 (1982) [JETP Lett. 36, 318 (1982)].

${ }^{28}$ P.A. Mello, P. Pereyra, and N. Kumar, Ann. Phys. (N.Y.) 181, 290 (1988).

${ }^{29}$ P.W. Brouwer, C. Mudry, B.D. Simons, and A. Altland, Phys. Rev. Lett. 81, 862 (1998).

${ }^{30}$ P.W. Brouwer, A. Furusaki, I.A. Gruzberg, and C. Mudry, Phys. Rev. Lett. 85, 1064 (2000).

${ }^{31}$ P.W. Brouwer, C. Mudry, and A. Furusaki, Phys. Rev. Lett. 84, 2913 (2000).

${ }^{32}$ F.J. Dyson, Phys. Rev. 92, 1331 (1953).

${ }^{33}$ T. Senthil and M.P.A. Fisher, Phys. Rev. B 60, 6893 (1999); S. Vishveshwara, T. Senthil, and M.P.A. Fisher, ibid. 61, 6966 (2000).

${ }^{34}$ O. Motrunich, K. Damle, and D.A. Huse, cond-mat/0011200 (unpublished).

${ }^{35}$ I. A. Gruzberg et al. (unpublished).

${ }^{36}$ T. Senthil and M.P.A. Fisher, Phys. Rev. B 61, 9690 (2000).

${ }^{37}$ The issue whether a generic disordered electron system belongs to one of Cartan's classes, or whether it requires fine tuning of the disorder distribution for a system to fall into one of the ten symmetry classes has been the subject of recent controversy in the literature (see Refs. 34, 20, and references therein). We do not want to add to this debate here. Instead, in this paper, our starting point is the assumption that the physical system at hand has a Hamiltonian belonging to one of the pure Cartan symmetry classes from Table I, for which the scaling equation for the transmission eigenvalues is given by Eq. (2), and our goal is to study the DOS singularity near zero energy under that assumption. With "pure" Cartan classes, we refer to those quantum wires whose Hamiltonian is taken from an ensemble of which the distribution is as random as possible within the bounds set by symmetry, or, alternatively, for which the motion of the transfer matrix in the appropriate symmetric space is isotropic (Refs. 3 and 22). Examples of Hamiltonians for the pure Cartan classes can be found in Refs. 29 and 30.

${ }^{38}$ C.W.J. Beenakker, J.C.J. Paasschens, and P.W. Brouwer, Phys. Rev. Lett. 76, 1368 (1996).

${ }^{39}$ N.A. Bruce and J.T. Chalker, J. Phys. A 29, 3761 (1996).

${ }^{40}$ V.I. Klyatskin and A.I. Saichev, Usp. Fiz. Nauk 162, 161 (1992) [Sov. Phys. Usp. 35, 231 (1992)]; C.W.J. Beenakker, K.J.H. van Bemmel, and P.W. Brouwer, Phys. Rev. E 60, 6313 (1999).

${ }^{41}$ M. Titov and C.W.J. Beenakker, Phys. Rev. Lett. 85, 3388 (2000).

${ }^{42}$ One reason why the stationary solution $P_{\varepsilon ; s t}$ is not of the simple form (7) is that the phases $\phi_{j}$ take values on the unit circle, which is not simply connected.

${ }^{43}$ A.A. Ovchinnikov and N.S. Érikhman, Zh. Éksp. Teor. Fiz. 73, 650 (1977) [Sov. Phys. JETP 46, 340 (1977)].

${ }^{44}$ H. Schmidt, Phys. Rev. 105, 425 (1957).

${ }^{45}$ M. Büttiker, J. Phys.: Condens. Matter 5, 9361 (1993).

${ }^{46}$ With the choice of the polynomials $p_{2 n-1}(\lambda)=\lambda^{2 N-n}+\lambda^{n-1}$ and $p_{2 n}(\lambda)=\lambda^{2 N-n}-\lambda^{n-1}$ the partition function can be written as a determinant of a matrix of size $N / 2[(N+1) / 2$ for odd $N]$. Then, the integration can be done analytically for $N=2$ and $N=3$

$$
\begin{gathered}
\left.Z(a)\right|_{N=2} \propto \frac{1}{a} \int_{2 a}^{\infty} K_{0}(t) d t, \\
\left.Z(a)\right|_{N=3} \propto \frac{1}{a}\left[K_{0}(a) K_{1}(2 a)-K_{1}(a) K_{0}(2 a)\right] .
\end{gathered}
$$

